\title{
La investigación responsable en los estudios de audiencia y recepción
}

\section{Responsible investigation in the audience and reception studies}

\author{
Mònica Figueras-Maz \\ LOREna Gómez-Puertas \\ Gema Revuelta \\ Universidad Pompeu Fabra \\ monica.figueras@upf.edu (ESPAÑA)
}

Recibido: 07.022018

Aceptado: 14.12 .2018

\section{RESUMEN}

La investigación responsable incluye, más allá de la ética en el contenido y el proceso, la implicación de múltiples actores y público, un acceso más fácil a los resultados científicos, la perspectiva de género, y la educación científica. El movimiento RRI (Responsible Research and Innovation) pretende que la investigación e innovación estén orientadas a conseguir resultados sostenibles, éticamente aceptables y socialmente deseables y que todo el proceso y las prácticas que comportan la investigación e innovación estén alineados con los valores, las necesidades y las expectativas de la sociedad. La investigación en comunicación, y específicamente, en audiencias y recepción, no se escapa de esta tendencia. En esta nueva concepción, se da un paso adelante respecto a la ética y se atribuye la responsabilidad no sólo al investigador sino que se avanza hacia el concepto de corresponsabilidad. En definitiva, con el impulso inicial de la Comisión Europea, se aboga por una investigación inclusiva y sostenible. Este artículo pretende, por un lado, plantear un breve repaso a la evolución y actualidad de los estudios de audiencia y recepción desde la perspectiva de las rupturas epistemológicas vividas y de los distintos retos a los que se enfrenta (conceptuales, metodológicos y éticos) y, por otro, entender el origen, evolución y tendencias del concepto de RRI para, finalmente, ver cómo puede aplicarse al estudio de la audiencia y recepción. El artículo tiene la vocación de convertirse en un texto que pueda ayudar a los investigadores del campo de la comunicación, y específicamente de las audiencias, a reflexionar sobre cómo poder incorporar la RRI a su trabajo. 


\title{
PALABRAS CLAVE
}

RRI, investigación responsable, audiencia, ética.

\begin{abstract}
Responsible research includes, beyond ethics in the content and process, engagement of multiple actors and the public, access to scientific results, gender perspective, and scientific education. The RRI (Responsible Research and Innovation) movement aims to create a society where research and innovation practices are oriented towards achieving sustainable, ethically acceptable and socially desirable results related to the values, needs and expectations of society. Research in communication, and specifically in audiences and reception, does not escape this trend. In this new conception, a step forward with respect to ethics is taken and the responsibility is attributed not only to the researcher but also to the concept of co-responsibility. In short, with the initial impulse of the European Commission, an inclusive and sustainable research is advocated. This article intends, on the one hand, to present a brief review of the evolution and actuality of audience and reception studies from the perspective of the epistemological ruptures experienced and the different challenges it faces (conceptual, methodological and ethical) and, on the other hand, to understand the origin, evolution and trends of the concept of RRI and, finally, to see how it can be applied to the study of the audience and reception. The article has the vocation to become a text that can help researchers in this field to reflect on how to incorporate the RRI into their work.
\end{abstract}

\section{KEY WORDS}

RRI, responsible research, audience, ethics.

\section{INTRODUCCIÓN}

En la última década y, muy especialmente, en los últimos cinco años, la Dirección General de Investigación de la Comisión Europea (CE) ha tenido un papel muy activo en la definición del concepto de Responsible Research and Innovation (RRI) y en su difusión. En el documento «Responsible Research and Innovation: From science in society to science for society, with society» (Comisión Europea, 2012), se resume la estrategia de la Comisión en este sentido. De hecho, el fomento de la RRI constituye una de las principales estrategias del programa marco Horizonte 2020 (H2020) de la CE y está integrado de manera transversal en los programas de trabajo anuales y las distintas convocatorias de financiación, de modo que es fácil entrever la capacidad de influencia que puede 
tener entre la comunidad investigadora. El documento «The Rome Declaration on Responsible Research and Innovation in Europe» es una buena prueba del avance en la difusión del concepto RRI entre la comunidad científica (SiS Conference, 2014).

Si bien la definición exacta de la RRI aún no está cerrada y existen diferentes matices en cuanto a su marco de implementación, el objetivo es, en todos los casos, introducir un cambio en la investigación e innovación para que éstas estén orientadas a conseguir resultados sostenibles, éticamente aceptables y socialmente deseables. Un cambio encaminado a que las prácticas y procesos propios de dicha investigación se alineen más estrechamente con los valores, las necesidades y las expectativas de la sociedad. El último programa marco de la Unión Europea para la investigación y la innovación (H2020) ha supuesto un estímulo para afrontar los retos global-local al plantear la RRI como un enfoque que anticipa y evalúa las implicaciones potenciales y las expectativas de la sociedad con respecto a la investigación y la innovación, con el fin de fomentar el diseño de una investigación e innovación inclusivas y sostenibles.

En la práctica, la RRI se ha estado implementando en proyectos de investigación de este programa marco como un paquete que incluye el compromiso de múltiples actores y público en la investigación y la innovación, permitiendo un acceso más fácil a los resultados científicos, la perspectiva de género, la ética en el contenido y el proceso de investigación y la educación científica formal e informal. Por citar algunos de los múltiples proyectos que han incorporado el enfoque RRI, tenemos los casos de $\underline{\text { RRI-ICTForum }}^{1}$ (centrado en el ámbito de las tecnologías de información y la comunicación), VOICES $^{2}$ (gestión de los residuos urbanos), NERRI (neuromejora) o SYNENERGENE $^{3}$ (biología sintética). Por otra parte, la Comisión Europea financia y promueve activamente herramientas y recursos para fomentar la expansión de la RRI. Entre otros, los proyectos $\underline{\mathrm{RRI}}$ ToolS $^{4}$ (una plataforma de recursos y redes que se dirige a múltiples sectores de la investigación), $\underline{\mathrm{HEIRRI}}^{5}$ (un proyecto para la integración de la enseñanza de la RRI en las universidades), o MORRI ${ }^{6}$ (un sistema de indicadores que permitirán seguir la evolución de la RRI y avanzar a partir de la comparativa entre países, organizaciones, etc.).

Así, el trasfondo de la RRI se plantea en muchos casos como base fundamental para el avance científico. En el Reino Unido, el EPSRC (o Consejo de Investigación en Ingeniería y Ciencias Experimentales) exige desde hace ya unos años que los proyectos que se presentan a las distintas convocatorias se desarrollen dentro de un marco de innovación responsable ${ }^{7}$. Y buena parte de los países miembros de la Unión Europea han recogido este enfoque en sus pro-

\footnotetext{
1 http://www.rri-ict-forum.eu

2 http://www.voicesforinnovation.eu

3 https://www.synenergene.eu

4 https://www.rri-tools.eu

5 http://heirri.eu

${ }^{6}$ http://www.technopolis-group.com/morri

7 https://www.epsrc.ac.uk/research/framework
} 
gramas y planes nacionales de investigación, innovación y desarrollo. En el caso de España, uno de los seis objetivos del Plan Estatal de Investigación Científica y Técnica y de Innovación 2017-2020 - en concreto, el Objetivo número $5^{8}$ - se centra precisamente en el compromiso con la investigación responsable y el acceso abierto al conocimiento, en línea con la definición de RRI de la Comisión Europea. Estos objetivos, pues, marcan los programas y subprogramas y las diversas convocatorias públicas de investigación e innovación.

La investigación en comunicación, y específicamente, en audiencias y recepción, no se escapa a esta tendencia. Si bien la ética es un aspecto fundamental en este campo de estudio porque se trabaja con seres humanos, la RRI incorpora otras dimensiones a la investigación. Además, respecto a la ética, en esta nueva concepción, se da un paso adelante y se pasa de atribuir la responsabilidad del investigador a una visión más amplia, de corresponsabilidad.

Este artículo pretende, por un lado, plantear un breve repaso a la evolución y actualidad de los estudios de audiencia y recepción desde la perspectiva de las rupturas epistemológicas vividas y de los distintos retos a los que se enfrenta (conceptuales, metodológicos y éticos) y, por otro, entender el origen, evolución y tendencias del concepto de RRI para, finalmente, reflexionar sobre su aplicación en el estudio de la audiencias y recepción. El artículo tiene la vocación de convertirse en un texto útil y propositivo que pueda ayudar a los investigadores de este campo a reflexionar sobre cómo poder incorporar la RRI a su trabajo. Si la finalidad de la RRI es ser útil a la sociedad, este texto coincide en cierto sentido con este propósito, ser útil a los investigadores en comunicación.

\section{LOS ESTUDIOS DE AUDIENCIA Y RECEPCIÓN: BREVE REPASO A SU EVOLUCIÓN Y TENDENCIAS}

La voluntad de este primer apartado es la de sistematizar algunos de los grandes ejes conceptuales que han marcado la evolución de los estudios de audiencia y recepción y cuya reminiscencia subyace a los debates que nos abre el campo de estudio de la comunicación actualmente y que tienen que ver con la responsabilidad de la investigación.

No se trata, por tanto, de explicar detalladamente los orígenes o las principales rupturas epistemológicas y políticas en la investigación de las audiencias desde principios del siglo XX, sino de situarnos en el estado de la cuestión en el que converge con un cambio de paradigma de la investigación social de mayor alcance.

${ }^{8}$ http://www.idi.mineco.gob.es/stfls/MICINN/Prensa/FICHEROS/2018/PlanEstatalIDI.pdf, pág. 31-32 


\subsection{De la masa a la agencia y a la investigación responsable}

La primera conceptualización de la audiencia como masa, más allá de la mal denominada 'teoría' de la aguja hipodérmica, incluye por un lado, las primeras aproximaciones con fines comerciales y por otro aquellas investigaciones de corte sociológico o empirista subyugadas a la idea de predecir el efecto directo deducible de la exposición al mensaje. Entre las primeras se hallan los primeros análisis cuantitativos para mapear a consumidores y votantes con una clara voluntad predictiva de su comportamiento. Entre las segundas surgirá un abanico diverso de aproximaciones, que abarca desde las bases de la Teoría del Cultivo de Gerbner a la Teoría fílmica británica basada en el análisis textual (Allen 1992). Junto a ellas, se trabajará a lo largo del siglo XX una mirada comprensiva y holística del poder presupuesto a los medios de comunicación de masas para el cambio social con una visión más o menos crítica, entre quienes parten de planteamientos próximos a la Escuela de Frankfurt y los denominados deterministas tecnológicos (Stevenson 1999). Obviamos aquí ciertas tentativas intermedias que bien pueden considerarse distanciadas de la concepción de la audiencia como masa, pero que han de observarse en tanto que propuestas que la investigación administrada sostiene desde la perspectiva funcionalista para cimentar la ideología liberal del ser humano autónomo y racional frente al débil efecto del sistema mediático y no como una clara orientación al análisis del contexto social de recepción (grupos de referencia y líder de opinión, hipótesis del refuerzo, variables de exposición selectiva o inclusive la teoría de la disonancia cognitiva) (véase: Brooker y Jermyn 2002; Nightingale 2011).

Un cambio substancial en esta evolución supuso considerar la audiencia como individuos, que dará lugar, desde una perspectiva normativista, a la línea de los usos y gratificaciones, y desde una perspectiva rupturista, a la reincorporación de la ideología y el poder de la mano de los Estudios Culturales, donde será clave el concepto de agencia (Morley 1992). En este nuevo paradigma de investigación de las audiencias se producirán diversas oscilaciones entre el poder del texto y el de la audiencia (véase: Grandi 1995), que parecen culminar con la denuncia del riesgo del relativismo extremo a finales de los noventa y la necesidad de estudiar holísticamente los procesos de sentido desde la producción a la recepción (Curran 1998). Así, la influencia en el campo de los Estudios Culturales anglosajones, incluyendo la vertiente crítica feminista, se evidencia en conceptos como representación, polisemia e interpretación, que resultan esenciales para evaluar las variables de identidad (género, raza, etnia, entre otras), la diversidad de lecturas y los procesos de codificación-decodificación y replantearse las competencias (textuales, genéricas o sociales) de los individuos que componen la audiencia, sus modos de consumo, más allá de los usos y gratificaciones que motiven su exposición, o sus posibles implicaciones o evaluaciones de los textos. En su desarrollo transcultural acorde a la evolución compleja de las sociedades globalizadas, los Estudios Culturales asientan premisas ineludibles hoy en el abordaje de las audiencias. Entre otras, destacamos cómo el placer se desvincula de la aceptación ideológica, al igual que la crítica de la idea de resistencia (véa- 
se: Barker 2000; Alatuusari 1999). Por otro lado, la evolución de los Estudios Culturales Latinoamericanos de la mano de Jesús Martín-Barbero y su teoría de las mediaciones, permite integrar nuevas y complejas aproximaciones al consumo interpretativo, los procesos de resemantización y su contextualización en diferentes niveles. Los enfoques etnográficos de análisis del proceso comunicativo desde su producción a su consumo y recirculación como experiencia del ciudadano de los que Thomas Tufte es referente, han consolidado el abordaje desde diseños multimetodológicos abiertos a la captación de la vivencia de las audiencias.

En definitiva, la ruptura respecto al paradigma anterior supone considerar la experiencia estética del receptor tanto individualmente como colectivamente, y revisar en profundidad las dicotomías que emergen en esta nueva aproximación a las audiencias (contextualización versus mediacentrismo; comunidades interpretativas y/o variables sociodemográficas; niveles micro o macro y teorías de las mediaciones; metodologías cuantitativas o cualitativas; interdisciplinariedad o transdisciplinariedad). Es en este cambio de paradigma donde empieza a cobrar sentido la investigación responsable en el sentido de atender a las necesidades de la sociedad y de explorar no sólo el papel de recepción de la audiencia (colectiva e individualmente) sino también su rol en el proceso de investigación. El centro ya no es el medio sino el receptor, su realidad y sus relaciones, pero se requiere de su colaboración consciente para construir e interpretar conocimiento sobre su actividad mediática.

Pero al hablar de cambios de paradigmas debemos ser cautelosos. Si algo ha caracterizado el siglo XXI es nuestra percepción de que todo cambia de modo acelerado, aunque quizá nada cambia excepto nuestro modo de observar. Ofrecer una revisión crítica del estado de la cuestión en el campo de la investigación de audiencias hoy en día es sumamente complejo y requiere de una actualización no sólo constante sino también amplia en cuanto a los objetos, los métodos y las perspectivas teóricas de análisis. Ontología, epistemología e ideología son tres ejes en permanente interacción para la definición de la comunicación contemporánea y por ende, del propio concepto de audiencias.

"In short, notwithstanding the conciliatory hopes of some (e.g. Blumler, Gurevitch, y Katz 1985), the different paradigms of audience research remain distinct and useful. Each raises particular questions, each instantiates a different conception of power, and each conjures a different vision of the audience -as an aggregate of individual consumers, as social collectives stratified by class, generation, gender, etc., or as individualised but not individual performers socially constituted through the shared performance of self. Arguably all paradigms continue to coexist because all roles for the audience coexist -could one even expect to settle on a single allencompassing view of people's mediated relation to the social?" (Livingstone 2013:7)

Nuestra voluntad aquí es la de recoger sintéticamente las reflexiones conceptuales y metodológicas que marcan algunas de las principales tendencias para el análisis de audiencias consensuadas por la comunidad científica. De ellas se derivan los retos y riesgos que afronta el campo de investigación académica, algunos 
no resueltos con anterioridad y otros redefinidos por el propio ecosistema mediático en el marco de cambios tecnológicos, organizacionales, culturales, políticos, económicos y sociales en el que se define y del que participa (Meyrowitz 1985; Thompson 1995). Otro reto que añadiríamos y que no proviene del propio ecosistema mediático son las consideraciones éticas en la investigación en cualquier campo y las políticas europeas que como se ha indicado vienen presionando en este sentido desde hace unos años.

"All of the above implies that we have to advance prudently in dealing with social change, and strike a balance between discontinuity and continuity, nonlinearity and linearity, homogeneity and diversity, critique, celebration and hope. (...) Audiencehood is intimately intertwined with our social realities..." (Carpentier et al. 2014:2)

La necesidad de contextualizar correctamente los cambios sociales que defiende Carpentier et al. (2014), y aquí compartimos, no es una novedad en la aproximación al estudio de la comunicación, si bien su visión antiesencialista del devenir de la humanidad aplicada al estudio de la audiencia nos permite tomar una posición distante y crítica frente a un objeto aparentemente voluble y cambiante. Para estos autores, aunque los nuevos y viejos medios de comunicación han cobrado protagonismo en estas últimas décadas a partir de las crecientes innovaciones tecnológicas, debe tenerse en cuenta que el auge de la participación mediática (o no) no puede desvincularse de procesos de democratización, comercialización, profesionalización e individualización tanto de la producción como del contenido, aplicables a otras tantas esferas o ámbitos de acción pública y privada. Los avances tecnológicos aplicados a la comunicación han facilitado o quizá intensificado estos procesos, pero no por ello puede atribuírseles su promoción, como tampoco pueden generalizarse las prácticas cross-media de la audiencia -cuya diversidad prevalece- ni establecerse rupturas inexistentes, puesto que no todos los procesos sociales son propensos al cambio, ni todos los cambios prevalecen.

Es en esta orientación de la investigación hacia la contextualización (entendida como inclusividad), la reflexividad (necesidad de cuestionarse las acciones y decisiones) y el compromiso con la sociedad, elementos propios de la investigación responsable, que cabe revisar la evolución de los estudios de audiencia desde la intervención sociopolítica. Se trata de un campo metodológicamente diverso, que a finales del siglo XX se estructuraba en dos áreas según el contenido de la comunicación educativa fuese intencional o no (Non-/Purposive Approach) (Fadul et al. 1993). Por un lado, en la comunicación prodesarrollo, el diseño estratégico de mensajes educativos-persuasivos con carácter socio-estructural destinado a países en vías de modernización recurre a estudios sociodemográficos para la observación de su eficacia (cambios de comportamiento o roles sociales). Por otro, en los denominados medios prosociales (o también conocidos como entretenimiento-educación) se valora el proceso intermedio o el consumo productivo de las audiencias a través de técnicas etnográficas que beben de la pionera línea de Martín-Barbero y otros autores emblemáticos de Latinoamérica 
(ver Servaes, 2002; Singhal et al. 2004), y más recientemente se han incorporado las técnicas propias de los estudios de los usos y efectos o la psicología de los medios sobre mensajes no intencionales, desde la óptica amplia que deroga cualquier compartimentación funcional de los mensajes mediáticos (véase, p. ej. sobre narrativa: Green et al. 2002; Igartua 2007).

Pese a todo, autores como Scott y Dietz (2016) denuncian la falta de investigación de audiencia en los países en vías de desarrollo y las consecuencias que esto supone tanto para la implementación de políticas públicas como para el correcto diseño de los proyectos destinados a integrar la comunicación mediática en los debates de gobernanza y las intervenciones socioeducativas. Esto conlleva realizar afirmaciones categóricas como que el acceso a internet empodera por sí mismo o supone efectos uniformes en la población, sin tener en cuenta la complejidad de los procesos de recepción y los usos políticos que de los medios puedan hacer los ciudadanos. Tras la revisión de la literatura académica y gris sobre este campo, estos autores recomiendan reorientar el estudio hacia la inclusividad desde la segmentación de la audiencia (atendiendo minorías, flujos y el grado de actividad/pasividad de los usuarios-ciudadanos) o la evaluación de la influencia de los medios en los contextos socio-culturales (desde la medición fiable de audiencias, a través de Joint Committees Industries hasta el recurso a triangulaciones metodológicas que permitan evaluar la confianza en la comunicación mediática frente a otros modos de interacción.

\subsection{Retos en la conceptualización de la audiencia y en los diseños metodológicos}

Afrontar el concepto de audiencia, como hemos podido recordar más arriba, ha sido un reto constante del campo de estudio de la comunicación (Abercrombie y Longhurst 1998). Actualmente prevalece la idea de una audiencia fragmentada y heterogénea para los medios de comunicación tradicionales, mientras que se acepta la idea de usuario (Livingstone, 2002 en Carpentier et al. 2014) como punto de partida adecuado para los nuevos medios. Aún así, como apuntan los editores de "Audiences Transformations" (compilación COST-Action ISO906) es un término que en sus derivaciones ante el intercambio de roles de producción y recepción que suponen los nuevos medios (produsers; prosumers) privilegia a los usuarios activos e ignora el consumo pasivo que pese a todo, sigue siendo audiencia.

Por otro lado, la necesidad de segmentar la audiencia para su análisis enfrenta la complejidad que las sociedades contemporáneas suponen en términos de transculturalidad y de disgregación de variables sociodemográficas explicativas de los usos y consumos de los medios de comunicación (etnia, género, edad, p.ej.) que habían sido aceptados en el paisaje comunicativo de antaño. La transmedialidad que suponen las diversas formas de incorporación de los nuevos medios (social media) por parte de las audiencias, supone afrontar nuevos retos metodológicos para su estudio (véase en la compilación de Patriarche 
et al. 2014, p.ej., los capítulos de Hight; Courtois et al.; Lemish y Nimrod; o Hujanen y Kangaspunta). Pero también retos conceptuales. En este sentido, cobra importancia la necesidad de explorar el porqué del uso o no uso de los social media, más allá del acceso (brecha digital), desde la clásica hipótesis de la alfabetización (Media Literacy) aplicada a la incesantemente variable oferta en red (Livingstone et al. 2014), o sencillamente el habitus, tal y como apuntan Trültzsch-Wijnen et al. (2015). Del mismo modo, cabe replantearse el concepto de agencia en un paradigma de individualización del produser de los social media. Aquí entran en juego la recuperación de la perspectiva interpretativa propia de Goffman y los interaccionistas simbólicos, al plantear conceptos como el yo imaginado, la reputación on/off-line percibida, el carácter autoreflexivo de la comunicación pública o las tensiones entre el grupo de afinidad y el de filiación (Aroldi y Vittadini 2015). Sin descuidar, en el marco de la cultura participativa de la que nos ocuparemos a continuación, la cuestión de la confianza aplicada a los propios medios de comunicación (Pavlícková et al. 2014) que constituye un elemento clave en la agenda política actual (en la línea que va de la desafección política de la ciudadanía a la era de la post-verdad).

La cultura participativa de los social media tal y como la describen autores como Jenkins desde el concepto convergencia o Scolari desde el transmedia (ver Jenkins et al. 2016), aúna el proceso de comercialización top-down con el del consumo y producción bottom-up que caracteriza al prosumer. Lo que entronca con la teoría de la audiencia activa como punto de encuentro del debate entre los procesos de significación e interpretación del campo de estudio precedente. Aún así, como se ha apuntado más arriba, no debe perderse de vista que dicha potencialidad sigue estando sujeta a (la falta de) acceso, competencias o habilidades (incluida media literacy) y la confianza de la que depende toda forma de participación e interacción comunicativa. El verdadero reto consiste, por tanto, en obtener información fiable sobre estas variables al analizar la interacción de la audiencia con la comunicación mediática.

Pero por lo pronto, el consenso más amplio entre la comunidad científica al plantearse este reto es el de la necesidad de innovar en el análisis de la audiencia a partir de diseños multimetodológicos. Pese a la clara divergencia entre la tradición cuantitativa (enfoque positivista, métodos estandardizados propios de las matemáticas extensivas e incorporación de las ciencias experimentales) y la tradición cualitativa (enfoque interpretativo, métodos etnográficos e incorporación de diversas disciplinas como la sociolingüística o la antropología), la superación en términos epistemológicos es un hecho contrastable ("Today, the methodological discussion is more pragmatic and less ideological, and researchers have started to think about how to use the respective strengths of the approaches to further insights (e.g. Baumann y Scherer 2012; Schroder et al. 2003, Teddlie y Tashakkori 1998)" (Patriarche et al. 2014: 5). Se trata, por tanto, de combinar metodologías de aproximación a los diferentes niveles de comunicación, a fin de complementar las fortalezas y/o paliar las flaquezas de los tradicionales enfoques cuantitativos y cualitativos (recuérdese la clásica regla proporcional entre lo manifiesto y observable y lo latente y relevante que ya planteaba en su origen 
el análisis de contenido). Así, tal y como nos indican estos autores, la estrategia común a las investigaciones contemporáneas de audiencia pasa por la diversificación e integración de métodos que permitan la triangulación, para una más profunda comprensión de los fenómenos comunicativos en un entorno mediático cada vez más complejo.

Unidas a estas reminiscencias propias de los conflictos abiertos por los Estudios Culturales a finales del siglo XX, se reabre con intensidad el reto de aproximar la realidad del investigador a la del sujeto o los sujetos investigados. Se trata de contextualizar, en este caso, la propia investigación superando el aumentado obstáculo que supone la individualización exacerbada de esta nueva era. Es justo en esta ruptura en los estudios de audiencia y recepción cuando podemos decir que se converge con el "espíritu" de la investigación responsable. Esto es, el individuo como centro pasa de ser un sujeto observado más o menos consciente a un sujeto integrado, reflexivo e iterativo, en el proyecto de investigación: desde la composición de los equipos y el diseño de los protocolos, hasta la interpretación y difusión de los resultados, como se verá en el apartado 2.

Antecedentes de este tipo de aproximación pueden encontrarse en el concepto de «investigación-acción» acuñado por Kurt Lewin en 1946, entendido como un proceso participativo y democrático llevado a cabo con la propia población local, de recogida de información, análisis, conceptualización, planificación, ejecución y evaluación. Se trataba de una propuesta que rompía con el mito de la investigación estática y defendía que el conocimiento se podía llevar a la esfera de la práctica, que se podían lograr de forma simultánea avances teóricos, concienciación y cambios sociales.

Más tarde, en los setenta y en Latinoamérica, se retoma la InvestigaciónAcción Participativa (IAP) con autores como Fals Borda (1987) e inspirados por Freire. Su elemento común y que coincide también con la RRI, es la preocupación sobre la utilidad de la investigación para la mejora de la sociedad (movimientos de renovación pedagógica, de intervención comunitaria...). El referente de la investigación tradicional es el de una persona o grupo experto (sujeto de la investigación) que aborda un aspecto de la realidad (objeto de la investigación) para comprobar experimentalmente una hipótesis, describirla o explorarla, sin que la comunidad sobre la que se hace el estudio, o para la cual se hace, tenga algún rol en el proceso más allá de poder conocer las conclusiones. En cambio, la IAP parte de otro enfoque de investigación científica donde se busca una mayor participación y apropiación del proceso y con ello también de los resultados por parte de la comunidad. Los miembros de las comunidades no son simples receptores o beneficiarios sino que actúan también como productores de conocimientos y se establecen relaciones horizontales orientadas a la transformación social.

Para contar con esta participación activa y performativa de la comunidad en la propia investigación que plantea la RRI y el precedente de la IAP, desde el ámbito de los estudios de las audiencias, los editores de la reciente compilación "Revitalising Audience Research" (COST-Action ISO906) consideran que deben implementarse metodologías y técnicas que asuman este carácter performativo y permitan acceder y capturar la experiencia cotidiana de los produser. A lo que se 
le añade la característica de iteratividad en el proceso, que ahonde en la reflexividad propia de la investigación responsable al convertir al sujeto investigado en el centro mismo del estudio. Es decir, en lugar de seguir la estela de las propuestas etnográficas (observación participante, grupos de discusión con intervención mínima del investigador, etc.) orientadas a eliminar o reducir la percepción de ser observados de los sujetos de la muestra, corresponsabilizarlos de la investigación llevada a término. Esto implica una reorientación en la que el investigado es el centro de la investigación de un modo consciente y contribuye a reducir la distancia con el investigador.

Creemos así, situar las variables de reflexividad e inclusividad de la RRI en la propuesta de aproximación a los estudios de la audiencia desde el actual paradigma de la participación. El punto de partida es común, el del construccionismo relacional, es decir, la realidad es construida a través de la interacción entre individuos reflexivos y conscientes. Y tal y como plantean Patriarche et al. (2014:6) esta premisa conlleva tres implicaciones metodológicas: 1) el conocimiento de la producción debe partir de las propias personas, sus narrativas de las situaciones y experiencias vitales, y no de las hipótesis del investigador; 2) los sujetos investigados son, así, informantes pero también co-analistas; y 3) por tanto, el análisis debe ser un proceso colectivo. Todo ello supone un reto significativo para la investigación tanto a nivel de recursos como de replanteamiento epistemológico y de asunción de cuestiones éticas. En cualquier caso, esta apertura metodológica que pone el foco en la necesidad de incorporar a los actores y redistribuir los roles clásicos, en la mayor transparencia de la investigación así como en la capacidad de adaptación, converge de forma clara con el cambio en el modelo actual de ciencia hacia la responsabilidad, como se verá después.

\subsection{El Big Data: dilemas éticos frente a los límites y las potencialidades}

Los denominados genéricamente social media (o Social Network Sites, SNS), incluyendo aquellos dedicados a crear y compartir contenidos y aquellos centrados en la socialización y conexión en red, permiten el acceso a bases de datos con los perfiles de usuarios tal y como están organizados en sus plataformas a través de las denominadas API (Application Programming Interface). El reto para la investigación de audiencias se desdobla entre el tratamiento de esta ingente cantidad de datos -asumido por la ingeniería informática que da origen a la "cultura algorítmica" - y la aportación que pueda llegar a hacer la academia desde la adaptación e integración de metodologías de investigación a este contexto. Parece obvio que tanto el acceso como el manejo del denominado Big Data recae en su dimensión más amplia en el sector industrial, que asume el coste cada vez más rentable del almacenamiento de la información y la minería de datos.

La investigación académica se ve relegada tanto por la escasez de recursos como por la inviabilidad de competir contra una explotación de alto valor comercial. A pesar de estas limitaciones, se abren múltiples posibilidades ante la 
investigación de audiencias a través de Big Data, y con ellas, más de un dilema ético a resolver.

Por un lado, la posibilidad de usar internet como herramienta de investigación ha sido objeto de debate tanto en la tradición cualitativa como en la cuantitativa (Jones 1999 en Patriarche et al. 2014). Estos mismos autores recogen el debate ético abierto en torno a la obtención de muestras, la fiabilidad de los datos obtenidos y los problemas de confidencialidad que supone partir de la producción y recolección de datos a través de la red (ver también, Boyd y Crawford 2012). Respecto al primer aspecto, sí es cierto que puede evitarse o minimizarse la percepción del sujeto de ser observado al recabar datos publicados en red, si bien cabe plantearse desde un punto de vista ético su uso no autorizado para fines de investigación. Más allá de este aspecto, la falta de transparencia del proceso de obtención de datos, priva a la investigación de su correcta contextualización, así como de la fiabilidad y significación que se obtendría de un proceso iterativo con el sujeto observado. Es por ello, que, a nuestro entender, queda abierta la cuestión de la representatividad de la audiencia, dada la limitación fehaciente de poder establecer una correlación entre la denominada audiencia social y la audiencia tradicional (esto es, entre la audiencia de los social media y la de los medios convencionales) (Claes y Deltell 2015).

Por último, algunas de las ideas aportadas por Adrian Athique (2018) sobre las potencialidades del Big data para la investigación de audiencias son esenciales para redimensionar el papel de la investigación académica futura. En primer lugar, la idea de falsedad aplicada a la "identidad digital" que imponen los social media, al combinar compras, seguimientos (fan), citas y conocidos (capital social) y CV (capital cultural) para la construcción de perfiles comercializables. Si bien se reconoce el potencial de este vasto conjunto de datos individualizados y en estructura relacional diseñados para la correlación entre comportamientos pasados y potenciales en relación a productos, actividades y acciones (Athique 2018) es obvio que se descuidan todos aquellos aspectos (y tantos otros) de los que se había ocupado la investigación de audiencias desde la perspectiva interpretativa y crítica. Correlación no es sinónimo de causalidad, aunque tal y como destaca Athique (2018:65-66) en su metafórico análisis entre la numerología y la alquimia de los metadatos: "Without having the slightest idea why people make those choices, a purely numerological correlation predicts behaviours on the basis of data patterns without worrying a jot about causality". No importa el por qué la gente hace lo que hace ahora que empresas y organizaciones cuentan con la posibilidad de predecir comportamientos. Y esto es así porque el usuario de los social media pasa a ser mercancía en la nueva economía digital, algo éticamente insostenible para el trabajo académico.

Es más, "más allá de las restricciones éticas que son antitéticas a la era del Big Data (Boyd y Crawford 2012)" (Athique 2018:67), la obtención de estos datos "sucios" o secundarios ya no pasa desapercibida a los usuarios, lo que restaría validez: "As users become aware of the pervasiveness of data trails, there is now an obvious "chilling" effect" (...) At a more general level, it is already evident that as our awareness of secondary data processing and the permanency 
of our user history have started to sink in, people are taking evasive action online (Andrejevic 2013)" (Athique 2018:67). Para el autor, esta razonable sospecha de sentirse escrutado actúa como revulsivo añadido ante el investigador académico que respeta las obligaciones éticas al requerir la colaboración de los sujetos investigados, que, como ya hemos señalado más arriba, es a día de hoy imprescindible en el contexto del construccionismo relacional y la cultura participativa. Así, no sólo se ve limitado a la arquitectura de los sistemas de los social media, sino también ve peligrar la obtención e integridad de los datos voluntarios.

A pesar de todo, en su exhaustiva y sistemática revisión de la investigación de audiencias en la era Big Data, Athique (2018) abre una brecha para el estudio académico. Y es que la investigación de audiencias es investigación social. Y al igual que las tendencias aquí resumidas emanan reminiscencias de los avances (y de los obstáculos) en la investigación de la audiencia desde sus orígenes, la "cultura algorítmica" "parece enfrentar de nuevo a los positivistas con la inconsistencia de esquemas para su interpretación, y a los investigadores de corte cualitativo, según el autor, con la negación de la lógica de la serialidad al interpretar las culturas. Ciertamente, afirma Athique (2018:74), “el énfasis predominante en la correlación y el reconocimiento de patrones sobre la integridad de datos puede ser adecuado para los estudios de comportamiento de compras con objetivos limitados, pero son cimientos inestables para cualquier comprensión de las culturas humanas". En todo lo que en definitiva pudiera acercarnos a las causas, en todo lo que nos hace humanos, aún hay mucho por aportar en el análisis -detallado, minucioso, "granular" en términos de Athique (2018)- del magma de los metadatos, como un reflejo más de las audiencias. Porque "mientras recordemos que las audiencias no son datos, todo debería ir bien" (Athique 2018:74). Y tal como iniciábamos este apartado, todo cambia o todo sigue, por lo que readaptamos nuestras aproximaciones al análisis de la realidad social desde el fenómeno integral de la comunicación.

En resumen, en la investigación de audiencias, debemos asumir los retos hasta aquí sintetizados (contextualización, cultura participativa, diseños multimetodológicos...) sin olvidar los conceptos clave y las eternas dicotomías que han estructurado la investigación de audiencias como investigación social (individuo/masa/grupo, interpretación/evaluación, agencia, apropiación, placer, resistencia motivación/implicación, identificación/proyección...) y el Big Data y sus implicaciones éticas. Vamos a ver en el apartado siguiente cómo además, aparece otro reto que es transversal a los anteriores pero que está cobrando cada vez más importancia en el debate sobre la investigación y la innovación actualmente a nivel europeo. Sin embargo, incorporado al estudio de la audiencia y

9 Reproducimos la definición de cultura algorítmica recogida por Athique (2018:69): ‘el uso de la computación de procesos para ordenar, clasificar y jerarquizar personas, lugares, objetos e ideas, y también los hábitos de pensamiento, conducta y expresión que surgen en relación con esos procesos' (Hallinan y Striphas 2016:119). 
recepción, supone una oportunidad para hacer mejor investigación y sobre todo, más implicada y reflexiva.

\section{EL NACIMIENTO DEL CONCEPTO RRI Y SU EVOLUCIÓN}

A pesar de haber sido acuñada recientemente, la expresión "Investigación e Innovación Responsables" se ha extendido con rapidez tanto en el ámbito institucional y político -especialmente en Europa- como en el estrictamente académico (Ribeiro, Smith y Millar 2017). La Comisión Europea ha sido particularmente activa y decisiva en la génesis y difusión de este concepto, elaborando su propia definición e introduciéndolo con fuerza tanto en sus documentos estratégicos como en las propias convocatorias de financiación en materia de Investigación, Innovación y Desarrollo (I+D+i) (Owen, Macnaghten y Stilgoe 2012; Angelaki 2016). En el último lustro, numerosos congresos, cursos, publicaciones, proyectos de investigación y actividades de índole diversa han incluido en su título esta expresión, ya sea en su forma extendida o en su acrónimo "RRI" (procedente del original en inglés Responsible Research and Innovation).

No hay duda de que lo que podríamos denominar como movimiento RRI está experimentado una evolución exitosa, pese a la cual no existe aún un consenso claro ni sobre su significado preciso ni sobre cómo implementarlo y, en su lugar, encontramos una miríada de definiciones y marcos (Ribeiro et al. 2017). Esta aparente disparidad, sin embargo, no es de extrañar, pues estamos ante un fenómeno emergente y en evolución. Lo que probablemente es más revelador es que las distintas definiciones coinciden en algunos elementos (Pellé y Reber 2013) o, como mínimo, en ciertos patrones (Rip 2014). Precisamente estos elementos compartidos son la expresión de un movimiento que busca un cambio del actual sistema de ciencia y tecnología que garantice un mayor compromiso con la sociedad y el entorno, una redistribución de los actores y sus roles, una necesidad de reflexión sobre los posibles impactos futuros de las decisiones presentes y una mayor transparencia y apertura en los procesos de I+D+i. La investigación en comunicación, y en concreto, en estudios de audiencia y recepción, no se escapa de este movimiento.

La definición o visión más citada sobre el concepto de RRI es la de Von Schomberg:

"Responsible Research and Innovation is a transparent, interactive process by which societal actors and innovators become mutually responsive to each other with a view to the (ethical) acceptability, sustainability and societal desirability of the innovation process and its marketable products (in order to allow a proper embedding of scientific and technological advances in our society)" (Von Schomberg 2013) 
La Comisión Europea utiliza una definición muy similar:

"Responsible Research and Innovation means that societal actors work together during the whole research and innovation process in order to better align both the process and its outcomes, with the values, needs and expectations of European society" (European Commision 2012)

Una de las aportaciones que, sin duda, más ha diferenciado la visión de la Comisión Europea respecto a la literatura académica sobre el tema es su definición de un marco de la RRI basado en seis elementos o aspectos temáticos clave: a) la participación del público (public engagement o PE); b) la ética en la investigación y la integridad ética; c) la educación científica (etapa escolar) y la promoción de la cultura científica (población adulta); d) el acceso abierto (a fuentes, datos y resultados); e) la promoción de la perspectiva de género en la $\mathrm{I}+\mathrm{D}+\mathrm{i}$ (en el doble sentido de reducir las diferencias en términos de recursos humanos y de aplicar el análisis de sexo/género a los contenidos de la investigación); y, finalmente, f) el cambio en la gobernanza del sistema de ciencia y tecnología (European Commision 2012).

Los seis aspectos clave identificados por la Comisión Europea como base de la RRI se han traducido, operativamente hablando, en estrategias, planes, programas y convocatorias de financiación en I+D+i. Dada la capacidad de influencia de esta institución sobre sus estados miembro, así como sobre las organizaciones, centros e investigadores que reciben financiación para sus proyectos, es de prever que este esfuerzo tenga un impacto en los próximos años.

En lugar de concentrarse en aspectos temáticos, como ha hecho la Comisión Europea, los autores que más han contribuido al estudio y difusión de la RRI en la literatura académica describen un marco operativo basado en dimensiones más transversales. Fundamentalmente, las dimensiones más comunes en las numerosas definiciones de la RRI son: a) la anticipación (necesidad de mirar hacia el futuro), b) la reflexividad (necesidad de cuestionarse las acciones y decisiones, incluyendo el cuestionamiento de lo supuestamente conocido), c) la inclusividad (necesidad de incorporar a los diferentes actores en todo el proceso, haciéndoles no sólo partícipes sino también corresponsables) y d) la capacidad de adaptación y respuesta durante el proceso de la I+D+i (Stilgoe, Owen y Macnaghten 2013).

La dimensión inclusividad (Stilgoe et al. 2013) y el aspecto clave public engagement de la Comisión Europea (European Commission 2012) son muy similares. Por otra parte, el uso que da la Comisión Europea al concepto public engagement coincide también con las definiciones del mismo que se encuentran en la literatura académica (Stilgoe, Lock y Wilsdon 2014; Rowe et al. 2008; Zwart et al. 2017). Public engagement significa fomentar la participación del público y de los diversos actores interesados (usuarios finales, grupos afectados, grupos interesados, público general) aumentando su contribución durante todo el proceso de la investigación. Esto es, estos múltiples actores no solamente deberían ser informados de los resultados de la investigación, sino que sus opiniones deberían ser más escuchadas, consultadas y tenidas en cuenta a la hora de tomar decisiones. En la forma de public engagement denominada "ciencia ciudadana" 
se promueven incluso acciones en las que el público no experto puede tener un papel activo durante el proceso de recogida de datos o en otras fases de la generación de nuevo conocimiento (Weitkamp 2016). El concepto de participación ciudadana, desde el punto de vista de la RRI, implica también que todos estos actores son corresponsables de lo que suceda con la investigación, de cómo se lleve a cabo, de las decisiones que se tomen, de cómo se use y de las consecuencias de todo ello.

\subsection{El concepto de responsabilidad de la RRI}

Una aportación innovadora del concepto RRI es su visión de la responsabilidad (o corresponsabilidad) no solo en su acepción más extendida que busca la identificación de los culpables de las consecuencias negativas (sentido punitivo de la responsabilidad), sino también en un sentido más positivo de perseguir el "bien", de no desaprovechar oportunidades, de explorar cuáles son las expectativas de los actores y públicos, de anticiparse, de actuar según los valores socialmente compartidos, etc. (Pellé y Reber 2013).

En la literatura académica no es una novedad la idea de que se está observando un cambio -más o menos radical- en el sistema de ciencia y tecnología (incluyendo aquí tanto las ciencias experimentales como las ciencias sociales). Una de las conceptualizaciones en la descripción de dicho cambio que más influencia ha tenido ha sido la diferenciación entre un Modo 1 y un Modo 2 en la producción del conocimiento (Gibbons et al. 1994; Nowotny, Scott y Gibbons 2003). Los autores afirmaban, hace más de veinte años, que en el sistema de ciencia y tecnología se estaba desarrollando un nuevo modelo de producción del conocimiento (Modo 2) que discurre en paralelo y de manera complementaria al modelo al que estamos más familiarizados (Modo 1). Según estos mismos autores, el nuevo modelo opera dentro de un contexto de aplicación en el que los problemas no se establecen dentro de un marco disciplinario. Es transdisciplinario, en lugar de mono o multidisciplinario. Además, implica la interacción estrecha entre muchos actores, a través del proceso de producción del conocimiento, lo que añade transparencia social. En este modelo, como consecuencia de los cambios, se utiliza una amplia gama de criterios en el control de calidad. Como resumen Hessels y Late, mientras que los principales atributos del Modo 1 son el contexto académico, la homogeneidad, la autonomía y el control de calidad tradicional (peer review); los atributos del Modo 2 son el contexto de las aplicaciones, la transdisciplinariedad, la heterogeneidad, la responsabilidad social/reflexividad y las nuevas formas de control de calidad (Hessels y Van Lente 2008). En esta misma revisión, los autores comparan el Modo 2 con otras conceptualizaciones del cambio en el sistema de ciencia y tecnología descritas en la literatura, tales como los modelos de ciencia posnormal, el modelo de la triple hélice, la ciencia posacadémica y la investigación estratégica.

El concepto de RRI se ha ido construyendo a partir de modelos como los descritos aquí, pero no solo han influido en su definición los trabajos realizados 
en el marco de la sociología y la política de la ciencia, sino que otras disciplinas han tenido también un gran peso en la noción actual del mismo. En el ámbito de la comunicación, el campo de investigación centrado en la comunicación científica ha sido muy activo en la construcción de este concepto, especialmente en el aspecto de poner en valor la importancia de la participación ciudadana o public engagement en las decisiones que tienen que ver con la investigación y los usos de sus aplicaciones (ver, por ejemplo, Bauer, Allum y Miller 2007; Bensaude Vincent 2014). Otras disciplinas y sectores que también han contribuido sustancialmente han sido la ética de la ciencia, los movimientos de ELSA (ethical, legal and societal aspects of science), la Responsabilidad Social Corporativa, los movimientos de acceso abierto al conocimiento (Open Access y Open Science), la denominada Community Based Research e investigación participativa, etc.

\section{LA INTEGRACIÓN DE LA INVESTIGACIÓN RESPONSABLE EN EL ESTUDIO DE AUDIENCIAS Y RECEPCIÓN}

Vistos los retos conceptuales, metodológicos y éticos que plantea el campo de estudio de las audiencias y la recepción, y su tendencia a converger con aquellos aspectos fundamentales que plantea la RRI, parece lógico y necesario alinear este concepto con el proceso de investigación I+D+i. En este apartado nos proponemos justamente analizar cómo integrar el concepto RRI en los estudios de audiencia y recepción llevando a cabo una revisión de todas las fases del proceso de investigación: desde la exploración de la realidad existente y la formulación de los problemas, hasta la definición concreta de los objetivos, el diseño de investigación, la metodología y la propia interpretación y difusión de los resultados.

Para realizar esta propuesta de alineación en términos prácticos se tendrán en cuenta las definiciones y los conceptos revisados en los apartados anteriores, y en concreto, las dimensiones descritas por Stilgoe et al., y los aspectos clave en los que se basa el marco descrito por la Comisión Europea. Así, se seguirán las fases del proceso de investigación en tres subapartados: 1) constitución del equipo de investigación, 2) definición concreta del problema y de los objetivos, la selección del diseño de estudio y la metodología, la recogida de datos y análisis de los mismos; y 3) interpretación de los resultados y difusión de los mismos.

Nuestro modo de entender cómo incorporar la RRI al estudio de audiencias y recepción aquí define cómo integrar una mirada o modus operandi más que una sistematización de protocolos a aplicar. Dada la casuística imponderable que nos ofrece el campo de estudio abordado aquí, no tendría sentido plantear esquemas en abstracto. Por ello, y con la voluntad de no reiterar las propuestas de reflexión que ofrece la RRI, transversales a todo el proceso de investigación, nos centraremos en algunas decisiones particulares, propias de cada fase. 


\subsection{Constitución del equipo de investigación}

La exploración de la realidad existente tendría un primer paso obvio que es el que situaría nuestro interés en el campo de estudio de las audiencias y la recepción. Y dada la naturaleza compleja de nuestras sociedades contemporáneas, muy probablemente nos situaremos en la intersección de dos o más áreas de la investigación.

Imaginemos, por ejemplo, que nuestro interés se orienta hacia la fiabilidad percibida sobre las diferentes fuentes de información en torno a la vacunación infantil o la lactancia materna, la influencia de los ambientes mediáticos obesogénicos sobre adolescentes o el aprendizaje informal a través de redes sociales digitales y su relación con la violencia machista o el consumo de opiáceos en menores. Cualquiera de estos puntos de partida implicaría la superposición de áreas o subcampos de estudio junto al de las audiencias y la recepción, ya sea por el ámbito (sanitario, educativo, familiar, etc.) o por los posibles segmentos de la sociedad implicados (adolescentes, menores y adultos, personal sanitario, etc.) en los que, a priori, podríamos prever que tienen lugar los procesos comunicativos a analizar.

Este ejercicio de anticipación se completaría si en lugar de imaginarnos el impacto a corto plazo que pueda tener nuestra investigación pensáramos en el conjunto de todos los campos de en los que se enmarca y en cuáles pueden ser las consecuencias de los avances que se produzcan en ellos. Así, más allá del impacto que la investigación pueda tener en nuestro reducido campo académico o sobre una decisión política local concreta a partir de informes técnicos derivados de nuestro trabajo, deberíamos plantearnos qué supondría para los estudios de comunicación y en el ámbito correspondiente. Además, convendría preguntarse en este punto inicial y a lo largo del proceso, cuáles serían las contribuciones de nuestra investigación para el conjunto de la sociedad y los actores implicados, teniendo en cuenta sus necesidades, valores y expectativas. Por ejemplo, en el caso de la vacunación de menores o la lactancia materna: las necesidades podrían incluir la cobertura sanitaria o la prestación social, la protección, la seguridad; se vincularía a valores éticos y morales, y a derechos y deberes individuales o colectivos; y reuniría expectativas, posiblemente, en torno a la salud, el bien común pero también la libertad y la autonomía de decisión.

Por todo ello, en esta primera fase del proceso de investigación, deberíamos plantearnos la conveniencia de constituir equipos de investigación multidisciplinares. En nuestro caso, no sólo especialistas en comunicación -obviando la naturaleza multidisciplinaria propia del campo y específicamente del estudio de audiencias y recepción que ha sido detallada en apartados anteriores- sino también expertos en esos otros campos de conocimiento sobrepuestos o integrados en la realidad que queremos observar (ética, política sanitaria, adolescencia, parentalidad, etc.).

Por otro lado, la idea de public engagement, en la RRI, implicaría que no sólo los investigadores deberían ser quienes planteen las preguntas y tomen decisiones, sino que los múltiples actores que forman parte del proceso I+D+i de- 
berían intervenir a lo largo de todas sus fases de desarrollo. ¿Podríamos integrar a otros actores en nuestro equipo de investigación? Por ejemplo, adolescentes prosumers en el campo de la imagen corporal (youtubers, instagramers y otros influencers en torno a conceptos de belleza, dietética y nutrición, etc.), asociaciones de padres y madres o de adolescentes que han sido víctimas de diversas problemáticas vinculadas a este ámbito (desde trastornos del comportamiento alimentario a acoso escolar o ciberacoso), representantes de las instituciones educativas y de ocio más frecuentadas por los jóvenes, representantes de la industria alimentaria y de la moda junto a sus publicistas y comunicadores... ¿individuos y/o grupos activistas o sensibilizados o aparentemente ajenos a estas cuestiones pero también receptores potenciales a los que se orienta la comunicación que construye ambientes mediáticos obesogénicos?

Como puede deducirse de este breve ejemplo de incursión inicial, un planteamiento de este tipo puede enriquecer el desarrollo y el alcance de la investigación, pero a su vez, conlleva riesgos o consecuencias no siempre beneficiosas. Así, por un lado, una mayor comprensión de los razonamientos e inquietudes de cada grupo o actor social vinculado al proceso de investigación I+D+i y, quizá, una mayor implicación y responsabilidad por parte de los mismos. Pero, por otro lado, es posible que también plantee riesgos como por ejemplo, las consecuencias derivadas de tener en el mismo grupo de investigación a sujetos con opiniones contrarias, probablemente radicalmente opuestas.

En el mismo sentido, al constituir el equipo de investigación ha de plantearse la representatividad de cada uno de los participantes desde las consideraciones éticas y de responsabilidad que, de acuerdo al concepto RRI, requieren de una reflexión en profundidad previa a la toma de decisiones. Así, por ejemplo, en el caso de las vacunas cabría plantearse qué nivel de responsabilidad en el proceso deberíamos permitir a un representante de un grupo de opinión muy minoritario -en España, por ejemplo, los padres que no vacunan a sus hijos representan menos de un $2 \%$ del conjunto de familias- frente a un representante de un campo de investigación con una fuerte evidencia y consenso científico. O bien, abordar cuestiones como la siguiente: ¿qué grado de responsabilidad debería tener en el equipo de investigación sobre las fuentes de información fiables en torno a vacunas un representante de la industria? Parece evidente que no puede obviarse su participación pero sí deberíamos ser conscientes de los retos éticos que han de guiar nuestras decisiones en esta fase de constitución del equipo de investigación $\mathrm{y}$ en todas las restantes.

La reflexividad que se propone de la RRI para cada una de las decisiones que requiere la investigación conjuga aquí un planteamiento flexible en la acepción de la capacidad de respuesta y adaptación a las situaciones que puedan presentarse en el transcurso del proceso. Pensemos, por ejemplo, que al iniciarse la investigación emerge como actor social significativo en el abordaje de la lactancia materna una red de blogueras o un movimiento contrario a esta práctica que consigue amplia cobertura mediática, ¿deberíamos ignorarlo porque no era relevante en el momento de configurar el equipo o el diseño de la investigación? 
Probablemente comporte dificultades pero convendría explorar formas de incluirlo en la investigación en el punto en que se encuentre.

Del mismo modo, el concepto inclusividad nos haría plantearnos si están representados en este equipo aquellos colectivos que por alguna circunstancia pudieran quedar marginados. Por ejemplo, en el caso del aprendizaje informal a través de redes sociales digitales y su relación con la violencia machista o el consumo de opiáceos en menores, deberíamos tener presentes aspectos básicos que hemos señalado previamente en el estudio de audiencias y recepción como retos conceptuales a la hora de definir a los usuarios de la comunicación. Así, la inclusión de adolescentes debería hacerse de acuerdo a variables sociodemográficas pero también de acceso que configuran la brecha digital en este segmento de la población. Es decir, contemplar indicadores sociales, económicos, culturales y/o de habitus que tienen que ver con el uso o no uso, de unas u otras redes sociales digitales, y que redundan a su vez en el tipo de media literacy o alfabetización, el nivel de agencia desarrollado como usuarios o el grado de confianza ya no sólo en estos medios sino en todos aquellos que componen el ambiente comunicativo transmedia en el que los y las adolescentes desarrollan su actividad de acuerdo a la cultura participativa subyacente. Sirva a modo de ejemplo: antes de dar por hecho que los y las adolescentes se mueven en Facebook y tomar esta red como referencia, hagamos la pregunta al propio usuario. Quizá nos sorprenda completar los datos estadísticos con la variable de motivación de uso que los lleva a moverse entre Facebook, Youtube, Instagram, Snapchat u otras aplicaciones como WattPad. Algo que, como hemos visto, aún no resuelve el Big Data dados los retos de validez que afronta (falsedad de identidad digital o desplazamiento de la causalidad por la correlación).

Del mismo modo, la perspectiva de género, en esta fase previa a la propia investigación, plantearía algunas reflexiones y decisiones: ¿es el equipo de investigación equilibrado en términos de género? ¿el género menos representado tiene al menos el $40 \%$ de los componentes? ¿se trata de un equilibrio en términos absolutos o también se produce en los distintos niveles de la cadena de mando? Y deberíamos añadir en el concepto de inclusividad esas otras variables que configuran la dicotomía entre privilegio y opresión en cada sociedad (clase, raza, religión, etc.) para no incurrir en lo que autoras como Kimberle Crenshaw (1989) han descrito como interseccionalidad o múltiple exclusión, propia de las sociedades modernas (por ejemplo, mujer afroamericana, musulmana de clase baja). Este aspecto ya ha sido recogido en el campo de estudio de las audiencias y la recepción al incorporar los Estudios Culturales no sólo la mirada ideológica en la representación, sino también las variables socioculturales de la audiencia y los contextos de recepción transcultural.

A menudo es imposible que el equipo de investigación sea tan multidisciplinar, tan inclusivo, tan paritario y tan amplio. Especialmente en proyectos limitados en sus recursos o en el tiempo. Antes de renunciar a estos aspectos, nos podríamos plantear incorporar las voces de los distintos actores y representantes en otros órganos (por ejemplo, consejos asesores, consultivos o deliberativos, con mayor o menor capacidad de decisión). Aquí entran en juego también las 
directrices que propone la Comisión Europea en relación a la gobernanza, y la gestión de esta participación integrada de sujetos diversos en el equipo de investigación. La llamada investigación basada en la comunidad (community based research), los laboratorios sociales (social labs) y algunos modelos de ciencia ciudadana (citizen science) son otros ejemplos de experiencias imaginativas para integrar a los distintos actores ya desde las primeras fases de la investigación.

Hemos de pensar también que la constitución del equipo de investigación conlleva que los participantes no son simplemente sujetos observados, sino que se integran plenamente en un proceso iterativo que los conmina a tomar consciencia del compromiso que adquieren como corresponsables -en diferentes formulaciones posibles- de la investigación de audiencias desde la RRI. En este sentido, podríamos plantearnos una formación mínima básica sobre cultura científica que preceda la firma de un compromiso de participación, del mismo modo que se requiere el consentimiento informado para la utilización de datos. Este aspecto de educación científica se desarrollaría de manera simultánea y vivencial por las personas que integren el equipo de investigación.

\subsection{Definición del problema, los objetivos, el diseño y la metodología}

La definición precisa del problema de estudio y de los objetivos de la investigación, ¿debería estar en manos solo del equipo de investigadores o también deberían participar los propios sujetos y comunidades investigadas? En este sentido, como hemos visto, los estudios de audiencia y recepción, especialmente aquellos que parten de diseños multimetodológicos para integrar aspectos cualitativos, actualmente ya adoptan este cambio de paradigma, por lo que el concepto public engagement estaría claramente integrado, desde una perspectiva de la RRI. Lo mismo podría suceder con las restantes decisiones acerca del diseño y el abordaje metodológico, en mayor o menor medida, si nos planteamos que investigadores e investigados actúen conjuntamente, siendo co-responsables, co-partícipes, co-creadores de conocimiento. Esto presupone, como ya ha sido señalado en los apartados anteriores, no partir necesariamente de preguntas o hipótesis de investigación ajenas al equipo que se ha conformado. De este modo, no sólo la exploración de la realidad, sino también los objetivos, y el modo de completarlos (esto es, la aproximación metodológica), son generados en la anticipación conjunta y reflexiva del equipo, que deberá proseguir de manera inclusiva y adaptativa a lo largo de todo el proceso de investigación.

Supongamos que se decide explorar el aprendizaje informal en redes sociales digitales en relación al consumo de opiáceos por parte de menores apuntando a una triangulación metodológica que incluya una fase cualitativa (por ejemplo, con grupos de discusión o focus groups) y otra cuantitativa (por ejemplo, mediante el uso de un cuestionario). La definición precisa de cuántos grupos se constituyen, cuáles son los criterios de inclusión-exclusión, cómo se hace la difusión, qué información se proporciona, qué tipo de técnicas garantiza la iteratividad entre los participantes, etc. debería considerar los diferentes aspectos y 
dimensiones de la RRI (ética, integridad científica, inclusividad, acceso abierto, etc.). Por otra parte, la propia investigación debería ser realizada en términos de sostenibilidad (por ejemplo, no consumir más recursos de los necesarios, no realizar más grupos o más entrevistas de las justificables, etc.).

La perspectiva de género implicaría aquí -además de una cuestión relativa al capital humano- una reflexión sobre el género y el sexo en el propio objeto de estudio. En este sentido, deberíamos plantearnos, por ejemplo, si los roles de género influyen en la recepción de la información, en la influencia de ésta o en las decisiones finales. Es decir, además de pensar en términos de equilibrio de género (en el equipo de investigación, en la muestra, etc.) deberíamos plantearnos también la integración de un análisis de sexo/género. Esto se evidencia claramente en los estudios de recepción en torno a problemáticas vinculadas a la imagen corporal cuando no conciben la perspectiva de género integrada en el proceso de investigación. En el ejemplo de la obesogenia que apuntamos como posibilidad, sería altamente probable que las relaciones entre los ideales corporales masculinos y femeninos apuntaran a otros conceptos como la vigorexia o la ortorexia... o sencillamente, que en el transcurso de la investigación debiéramos replantear el binomio masculino/femenino.

El proyecto debería garantizar también la integridad científica a lo largo del proceso, estableciendo las medidas oportunas para que este se realice de la manera más responsable posible, evitando el fraude científico, el falseamiento de datos, la ocultación de datos negativos o inesperados, el abuso de poder dentro del equipo o con los participantes, etc. Si el proyecto se realiza en una institución o en un campo en el que no existe un claro marco sobre estas cuestiones, valdría la pena considerar cómo garantizar dicha integridad. Esto es altamente relevante en los ejemplos de estudios de audiencia y recepción planteados aquí, no sólo por la implicación de personas, y concretamente de menores, sino por cuanto los datos recogidos afectan a la identidad social de los sujetos, aspecto amplificado cuando se trata con redes sociales digitales.

Frente a la necesidad de garantizar la integridad científica -lo que nos lleva de nuevo a esta idea de educación científica a lo largo del proceso de investigación para los miembros del equipo- nos hallaremos con la necesidad de construir un proyecto sensible (a las recomendaciones de los participantes, a los cambios que se puedan producir en el entorno, etc.) y disponer de sistemas de respuesta y adaptación. Esta necesidad de atender al entorno y adaptarse al mismo debe estar prevista en el propio proyecto de investigación, de manera que se tracen aquellos límites imprescindibles que no se pueden transgredir y se compartan con todo el equipo desde el inicio.

\subsection{Interpretación de los resultados y difusión}

La recogida de datos, su tratamiento y difusión, más o menos abierta y transparente, sería otro de los aspectos a considerar. Deberíamos decidir aquí si se van a compartir todos los ficheros de datos, sus productos secundarios, los 
programas que hayamos creado o utilizado para la recogida o tratamiento de los mismos, etc. No se nos escapa que aquí se deben tener en cuenta no sólo las recomendaciones políticas actuales de ciencia abierta (open science), sino que nuestros datos tendrán que estar también sujetos a las consideraciones éticas propias de las leyes de propiedad intelectual, a los aspectos éticos que tienen que ver con la privacidad y la dignidad, etc. Sin embargo, se ha de consensuar un modo de proceder que garantice que el análisis y la interpretación de los resultados se realiza de manera corresponsable con el equipo de investigación. Por ejemplo, ¿podríamos plantear sesiones de trabajo conjuntas entre adolescentes y demás actores sociales implicados en el abordaje de la violencia machista a partir de la interpretación de los datos sobre el consumo y la recepción en redes sociales digitales? ¿Podría ser está sesión abierta e incorporarse a su vez como datos recabados?

En efecto, tocamos en esta fase también la complejidad que la RRI supone a la difusión de los resultados de la investigación sobre audiencias y recepción. El potencial altamente sensible de los datos y resultados a obtener en cualquiera de los ejemplos de estudio apuntados aquí, implica tomar decisiones acerca de la idoneidad de cómo compartirlos. La RRI propone también una difusión pública abierta y transparente (pueden ser resúmenes, textos divulgativos, acciones específicas para dar a conocer los resultados o las recomendaciones que puedan derivar de ellos, etc.). Por otro lado, en la transferencia en el ámbito científico debe garantizarse el acceso abierto (open access), pero incluso así, deberíamos decidir si este será en modalidad gold o green. Y cabe tener en cuenta es la autoría de los artículos, una cuestión que a menudo lleva a irregularidades y a abusos de poder. Garantizar la integridad científica en esta fase es tanto o más importante que en las anteriores.

Pero más allá de la transferencia en el ámbito científico, la RRI requiere también de esa otra transferencia más social, no solo acciones para la ciudadanía sino sobre todo con ella. Por ejemplo, la organización de charlas en escuelas junto con las asociaciones de padres y madres sobre el uso de redes sociales digitales y diversas conflictividades como las apuntadas en torno a la identidad, la imagen corporal, la violencia o el consumo de sustancias tóxicas. Los resultados generados a partir de estudios de audiencia o recepción podrían plantearse a modo de recomendaciones que pudieran servir de ayuda a educadores formales e informales o bien ser utilizado directamente por las familias. Del mismo modo, podría operarse en el ámbito sanitario, mediante acciones performativas en los centros de asistencia primaria, donde prevalece el contacto más íntimo entre personal médico y pacientes. Imaginemos una sesión de lectura de las revistas de familia y sus discursos en torno a la alimentación infantil como taller para futuras (o recientes) madres y padres en el que participen diversas voces. Esto recala, finalmente, en el concepto educación científica, fundamental para la Comisión Europea sobre la RRI. La investigación debería servir también para alfabetizar y formar ciudadanos competentes en términos de cultura científica y hacerles corresponsables del rumbo que tome la investigación, así como del uso que se le dé a las aplicaciones derivadas de ésta. 


\section{REFLEXIONES FINALES}

Los estudios de audiencia y recepción, como se ha visto, han pasado por muchas rupturas epistemológicas pero quizás la fundamental ha sido el cambio de paradigma del pasar del foco en el medio de comunicación o la masa anónima al contexto y a la agencia de los espectadores. Paralelamente, la manera de hacer investigación en todos los campos del conocimiento, es decir, la manera de hacer ciencia, también ha cambiado y está evolucionando hacia un paradigma en el que la ciudadanía y la sociedad son el centro de la investigación y, en este sentido, podemos decir que son dos procesos que convergen. El engagement y la corresponsabilización de la ciudadanía en el proceso investigador es requisito fundamental para la RRI.

Como hemos visto, no hay una definición única sobre qué es la RRI, pues es un concepto en evolución pero, en cualquier caso, la investigación responsable, sea con este nombre o con otro, es una aproximación necesaria e inevitable en el futuro de la ciencia en cualquier disciplina; los estudios de audiencia no se escapan de ello. Más allá de la ética como requisito fundamental en toda investigación con seres vivos, este nuevo paradigma supone un cambio de mirada para poder integrar de manera transversal otras dimensiones a las que quizás hasta ahora no se les había prestado quizás suficiente atención. El estudio de audiencias y recepción, cuyo foco son las personas, requiere además un cambio más profundo e integral en el abordaje de las investigaciones donde la anticipación, reflexividad e inclusividad sean ejes fundamentales. Y en el que la corresponsabilidad de los sujetos -ya no observados, sino necesariamente integrados en la investigación- permita adaptarse y dar respuesta a los retos conceptuales que plantea el cambio. Visto así, el consentimiento informado de los participantes pasa a ser una gota en el océano.

En el campo de estudio de las audiencias y la recepción se cuenta, desde ya hace tiempo, con un amplio abanico de metodologías y técnicas de investigación que permiten su abordaje interdisciplinar. No tanto así, y de ahí el reto, en su plena triangulación cuantitativa-cualitativa, dificultada por la falta de recursos y el entorno altamente sensible y cambiante de la cultura participativa y transmedial que se ha instaurado en las sociedades globalizadas. Pese a ello, no debe verse en este enfoque un intento de mercantilización u optimización de los participantes como proveedores y co-analistas de sus propias prácticas comunicativas. Contrariamente a este planteamiento, y tal y como hemos expuesto, la perspectiva RRI permite alinear estrechamente cada una de las fases y procesos propios de la investigación con los valores, las necesidades y las expectativas de la sociedad. Es la ciencia con y para la sociedad, y debe ser desde esta vocación desde la que se plantee.

En el mismo sentido la investigación responsable en el campo de los estudios de audiencia y recepción no es tampoco asimilable a la idea de generar estrategias comunicativas o de relaciones públicas en torno a temas o con la pretensión de crear una buena imagen de actores o instituciones de la índole que sea. Por definición, parte de la sociedad y retorna a ella, compensando desde los concep- 
tos de gobernanza, acceso abierto, educación científica, ética y public engagement, justamente cualquier uso fraudulento a priori.

Aunque se trata de un movimiento muy reciente que ha emergido sobre todo en el campo de las ciencias experimentales y de la salud, rápidamente se está extendiendo a todas las disciplinas de las ciencias sociales. La integración de la RRI (en todos o en algunos de sus aspectos y dimensiones) en el estudio de audiencias y recepción que aquí se ha planteado desde una voluntad exploratoria y propositiva, podría contribuir a mejorar la calidad de este campo de la investigación y potenciar su impacto científico y, sobre todo, social y comunitario.

\section{BIBLIOGRAFÍA}

ABERCROMBIE, N., y LONGHURST, B. (1998): Audience and sociological theory of performance and imagination, London, Sage

ALATUUSARI, P. (ed.) (1999): Rethinking the media audience: the new agenda, London, Sage.

ALLEN, R.C. (1992): “Audience-Oriented Criticism and Television”, en R.C. ALLEN (ed.) Channels of Discourse, Reassembled. Television and contemporary criticism, 2a ed., pp. 101-136, London, The University of North Carolina

ANGELAKI, M. (2016): An Introduction to Responsible Research and Innovation, disponible en http://pasteur4oa.eu/sites/pasteur4oa/files/resource/RRI_POLICY BRIEF. pdf [consulta: 15-1-2018]

AROLDI, P. y VITTADINI, N. (2014): “Audiences as Socio-Technical Actors: The 'Styles' of Social Network Site Users”, en ZELLER, F., PONTE, C. y O'NEILL, B. J. (eds.) Revitalising Audience Research. Innovations in European Audience Research, New York y London, Routledge

ATHIQUE, A. (2018): "The dynamics and potentials of big data for audience research", Media, Culture \& Society, 40(1), pp. 59-74

BARKER, C. (2000): Cultural Studies: Theory and Practice. London, Thousand Oaks y New Delhi, Sage

BAUER, M. W., ALLUM, N., y MILLER, S. (2007): "What can we learn from 25 years of PUS survey research? Liberating and expanding the agenda", Public Understanding of Science, 16(1), pp. 79-95

BENSAUDE VINCENT, B. (2014): "The politics of buzzwords at the interface of technoscience, market and society: The case of 'public engagement' in science", Public Understanding of Science, 23(3), pp. 238-253

BOYD, D., y CRAWFORD, K. (2012): "Critical Questions for big data: provocations for a cultural, technological and scholarly phenomenon", Information, Communication \& Society, 15(5), pp. 662-679

BROOKER, W., JERMYN, D. (2002): The Audience Studies Reader, New York y London, Routledge

CARPENTIER, N., SCHRODER, K.C., y HALLETT, L. (eds.) (2014): Audience Transformations. Shifthing Audience Positions in Late Modernity, New York y London, Routledge

CLAES, F. y DELTELL, L. (2015): “Audiencia social en Twitter: hacia un nuevo modelo de consumo televisivo", Trípodos, 36, pp. 111-132 
COMITÉ DE BIOÉTICA DE ESPAÑA (2016). Cuestiones ético-legales del rechazo a las vacunas y propuestas para un debate necesario. Madrid, Comité de Bioética de España. Disponible en http://assets.comitedebioetica.es/files/documentacion/es/ cuestiones-etico-legales-rechazo-vacunas-propuestas-debate-necesario.pdf [consulta: 15-1-2018]

CREENSHAW, K. (1989). "Demarginalizing the Intersection of Race and Sex: A Black Feminist Critique of Antidiscrimination Doctrine, Feminist Theory and Antiracist Politics." University of Chicago Legal Forum (1989), p. 139

CURRAN, J. (1998): "El nuevo revisionismo en los estudios de comunicación: una revaluación" en J. Curran, D. Morley, V. Walkerdine (eds.). Estudios culturales y comunicación (1a ed.), Barcelona, Paidós (trad. cast.. London: Arnold, 1996), pp. 383-415

EUROPEAN COMMISION (2012): Responsible Research and Innovation, Europe's Ability to Respond to Societal Challenges, disponible en http://ec.europa.eu/research/science-society/document library/pdf 06/responsible-research-and-innovation-leaflet en.pdf [consulta: 15-1-2018]

FADUL, A.M. (comp.) (1993): Serial fiction in TV. The Latin American telenovelas, San Pablo, ECA-USP.

FALS BORDA, O. y RODRÍGUEZ BRANDAO, C. (1987): Investigación Participativa, Montevideo, La Banda Oriental

GIBBONS, M., LIMOGES, C., NOWOTNY, H., SCHWARTZMANS, S., SCOTT, P., y TROW, M. (1994): The new production of knowledge : the dynamics of science and research in contemporary societies, London, Sage

GRANDI, R. (1995): Texto y Contexto en los medios de comunicación de masas, Barcelona, Bosch

GREEN, M.C., STRANGE, J.J., BROCK, T.C. (eds.) (2002): Narrative impact: social and cognitive foundations. Mahway, New Jersey, Lawrence Erlbaumm Associates

HALLINAN, B. y STRIPHAS, T. (2016): Recommended for you: The Netflix Prize and the production of algorithmic culture", New Media \& Society, 18(1), pp. 117-137

HESSELS, L. K., y VAN LENTE, H. (2008): "Re-thinking new knowledge production: A literature review and a research agenda", Research Policy, 37, pp. 740-760

IGARTUA, J.J. (2007): Persuasión narrativa. El papel de la identificación con los personajes a través de las culturas, Alicante, Club Universitario

JENKINS, H. MIZUKO, I. y BOYD, D. (2016): Participatory Culture in a Networked Era, Cambridge, Polity Press

LEWIN, K. (1946): "Action research and minority problems", Journal of Social Issues, 2 (4): $34-46$

LIVINGSTONE, S. (2013) "The participation paradigm in audience research", Communication Review, 16 (1-2), pp. 21-30

LIVINGSTONE, S., WIJNEN, C.W., PAPAIOANNOU, T., COSTA, C. y GRANDÍO, M. (2014): "Situating Media Literacy in the Changing Media Environment: Critical Insights from European Research on Audiences" en CARPENTIER, N., SCHRODER, K.C., y HALLETT, L. (eds.) Audience Transformations. Shifthing Audience Positions in Late Modernity, New York y London, Routledge

MARTÍN-BARBERO, J. (1987): De los medios a las mediaciones. Comunicación, cultura y hegemonía. Barcelona: Gustavo Gili

MEYROWITZ, J. (1985): No sense of Place: The impact of electronic media on social behaviour, New York, Oxford University Press

MORLEY, D. (1992): Television, Audiences and Cultural Studies, London, Routledge 
NIGHTINGALE, V. (ed.). (2011): The handbook of media audiences, Malden, WileyBlackwell

NOWOTNY, H., SCOTT, P., y GIBBONS, M. (2003): “ 'Mode 2' Revisited: The New Production of Knowledge", Minerva, 41, pp. 179-194

OWEN, R., MACNAGHTEN, P. y STILGOE, J. (2012): "Responsible Research and Innovation: From Science in Society to Science for Society, with Society", Science and Public Policy, 39(6), pp. 751-760.

PATTRIARCHE, G., BILANDZIC, H., JENSEN, J.L. y JURISIC, J. (EDS.) (2014): Audience research methodologies. Between Innovation and Consolidation, New York y London, Routledge

PAVLÍCKOVÁ, T., NYRE, L. y JURISIC, J. (2014): "What Does It Mean to Trust the Media?" en CARPENTIER, N., SCHRODER, K.C., y HALLETT, L. (eds.) Audience Transformations. Shifthing Audience Positions in Late Modernity, New York y London, Routledge

PELLÉ, S. y REBER, B. (2013): Governance of Responsible Innovation GREAT 321480 DEL.2.2. Theoretical Landscape, disponible en http://www.great-project.eu/ deliverables files/deliverables03 [consulta: 15-1-2018]

RIBEIRO, B.E., SMITH, R.D. y MILLAR, K. (2017): “A Mobilising Concept? Unpacking Academic Representations of Responsible Research and Innovation”, Science and Engineering Ethics, 23(1), pp.81-103, disponible en http://link.springer. com/10.1007/s11948-016-9761-6 [consulta: 15-1-2018]

RIP, A. (2014): “The Past and Future of RRI”, Life Sciences, Society and Policy, 10(1), disponible en http://lsspjournal.springeropen.com/track/pdf/10.1186/s40504-0140017-4? site=lsspjournal.springeropen.com [consulta: 15-1-2018]

ROWE, G., HORLICK-JONES, T.E., WALLS, J., POORTINGA, W. y PIDGEON, N.F. (2008): "Analysis of a Normative Framework for Evaluating Public Engagement Exercises: Reliability, Validity and Limitations", Public Understanding of Science, 17(4), pp. 419-441

SCOTT, M. y DIETZ, C. (2016): "From the Field: Bring back the Audience: A discussion of the Lack of Audience Research in the Field of Media Development", Global Media Journal, 6(2), pp. 1-7

SERVAES, J. (ed.) (2002): Approaches to Development Communication, París, UNESCO, disponible en http://www.unesco.org/fileadmin/MULTIMEDIA/HQ/CI/ CI/pdf/approaches to development communication.pdf [consulta: 15-1-2018]

SINGHAL, A., CODY, M. J., ROGERS, E. M., y SABIDO, M. (eds.) (2004): Entertainment-education and social change: History, research, and practice, Mahwah, New Jersey, Lawrence Erlbaum

SiS CONFERENCE 2014. (2014) "Rome Declaration on Responsible Research and Innovation in Europe." N.p., disponible en https://ec.europa.eu/research/swafs/pdf/ rome declaration RRI final 21 November.pdf [consulta: 15-1-2018]

STEVENSON, N. (1999): Culturas mediáticas. Teorías social y comunicación masiva, Amorrortu

STILGOE, J., LOCK, S.J. y WILSDON, J. (2014): "Why Should We Promote Public Engagement with Science?", Public Understanding of Science, 23(1), pp. 4-15.

STILGOE, J., OWEN, R. y MACNAGHTEN. P. (2013): "Developing a Framework for Responsible Innovation.” Research Policy 42(9):1568-80. Retrieved January 15, 2018 (http://linkinghub.elsevier.com/retrieve/pii/S0048733313000930)

THOMPSON, J. B. (1995): The Media and Modernity: A Social Theory of the Media, Stanford University Press. 
TRÜLTZSCH-WIJNEN, C., TRÜLTZSCH-WIJNEN, S. y SIIBAK, A. (2014): “Using and Not Using Social Media: What Triggers Young People's Practices on Social Network Sites?”, en ZELLER, F., PONTE, C. y O’NEILL, B. J. (eds.) Revitalising Audience Research. Innovations in European Audience Research, New York y London, Routledge

TUFTE, Th. (2015): Comunicación para el cambio social: participación y empoderamiento como base. Barcelona: Icaria.

TUFTE, Th. (2000): Living with the Rubbish Queen: Telenovelas, Culture and Modernity in Brazil. London. University of Luton Press

VON SCHOMBERG, R. (2013): “A Vision of Responsible Innovation”, OWEN, M. Responsible Innovation. Managing the responsible emergence of science and innovation in society, 3 , pp. 51-74

WEITKAMP, E. (2016): "From Planning to Motivations: Citizen Science Comes to Life", Journal of Science Communication, 15(3), disponible en https://jcom.sissa.it/ sites/default/files/documents/JCOM 15032016 E.pdf [consulta: 15-1-2018]

ZELLER, F., PONTE, C. y O'NEILL, B. J. (eds.) (2015): Revitalising Audience Research. Innovations in European Audience Research, New York y London, Routledge

ZWART, H. et al. (2017): "Reflection as a Deliberative and Distributed Practice: Assessing Neuro-Enhancement Technologies via Mutual Learning Exercises (MLEs)", NanoEthics, 11(2), pp. 127-138 